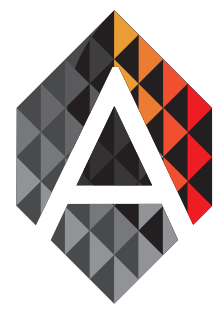

ADCAIJ: Advances in Distributed Computing and Artificial Intelligence Journal

Regular Issue, Vol. 7 N. 2 (2018), 13 -25

eISSN: $2255-2863$

DOI: http://dx.doi.org/10.14201/ADCAIJ20187213 25

\title{
Modeling of Circadian Rhythm under influence of Pain: an approach based on Multi-agent Simulation
}

\author{
Stefânia da Silveira Glaesera , Angélica Theis dos Santos ${ }^{\mathrm{a}}$, \\ Adriano De Cezaro ${ }^{a}$, Catia Maria dos Santos Machado ${ }^{a}$, \\ and Diana Francisca Adamatti ${ }^{a}$ \\ aPrograma de Pós Graduação em Modelagem Computacional (PPGMC) - Universidade Federal do Rio \\ Grande (FURG) Caixa Postal 474 - 96.203.900 - Rio Grande - RS - Brasil stefaniaglaeser@gmail.com, \\ theisangelica@gmail.com, adrianodecezaro@furg.br, catiamachado@furg.br, dianaadamatti@furg.br
}

$\begin{array}{ll}\text { KEYWORD } & \text { ABSTRACT } \\ \text { Multi-agent; } & \text { The human body has endogenous biological rhythms. One of those rhythms is the cir- } \\ \text { Circadian } & \text { cadian rhythm, with a cycle of approximate } 24 \text { hours, that is responsible for regulating } \\ \text { Rhythm; Pain } & \text { many important functions of human body. In this contribution, we propose a nonlinear } \\ & \text { coupled differential equation for modelling some agents responsible for the circadian } \\ & \text { rhythm under the influence of pain. We show results concerning the well-posedness } \\ \text { of a solution as well as an analysis on the synchronization for each agents. We use a } \\ \text { multi-agent simulation strategy of an corresponding model as an alternative interpre- } \\ \text { tation of the pain influences in the behavior of the circadian rhythm. }\end{array}$

\section{Introduction}

Chronobiology is a branch of biological sciences that aims to study biological, physiological and psychological phenomena, allowing the understanding that the human organism is composed of biological rhythms and cycles. The circadian rhythm is 24-hour biological rhythms that regulate important functions of the human body. In this approach, we consider two such rhythms, mainly the sleep-wake cycle and sleep pressure and how they react in the presence of disturbances, such as pain. To that end, we will see each rhythm under investigation as an agent that interacts with others. Therefore, we can simulate the actions and reactions of each part under investigation as a multi-agent strategy.

Multi-agent simulation is widely used today to assist in solving problems that are difficult to be solved analytically. This simulation is characterized by the existence of autonomous agents that work among themselves to meet a purpose. We will use the multi-agent simulations to explore insight into the collective behavior of the circadian rhythms as the agent, obeying a nonlinear coupled rule. See section 2.2. and section 2.2.1.

This paper presents two main novelties: First, we propose a coupled nonlinear oscillator dynamics for modeling inter-interaction between the sleep-wake and the corporal temperature cycle under the influence of an external agent, that we interpret as the pain. This is an extended version of the model proposed by Strogatz (Strogatz, 2013). We study the existence of solution for the proposed problem as well as present the solutions

Stefânia da Silveira Glaeser, Angélica Theis dos Santos, Adriano De Cezaro, Catia Maria dos Santos Machado, and Diana Francisca Adamatti

Modeling of Circadian Rhythm under influence of Pain:

an approach based on Multi-agent Simulation

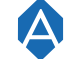

ADCAIJ: Advances in Distributed Computing and Artificial Intelligence Journal Regular Issue, Vol. 7 N. 2 (2018), 13 -25 elSSN: 2255-2863 - http://adcaij.usal.es Ediciones Universidad de Salamanca - CC BY NC DC 
for the synchronized and unsynchronized versions, based on the coupling strangest. Such simulated solutions shows the existence of delays in the synchronization when the pain acts in the system. Secondly, we use the above described mathematical based modeling to propose corresponding modifications in the multi-agent simulation approach that describes the rhythms proposed by (Skeldon, 2014) involving the pain as an extra valiable in the agents. The multi-agent simulations again shows the same delay synchronized characteristics when pain acts in the system. An important consequence of this study is to understood in with conditions the circadian rhythm returns into normality conditions after an external influence, i.e., the synchronization.

This paper is structured in four sections: In the first section, we present an introduction of the work. In the second section are conceptualized the circadian and homeostatic rhythms, and synchronization. We also present the circadian rhythmical models with the pain influences as a modification of Strogatz's and Borbély's models, for with we discuss the characteristics of the corresponding solutions. Furthermore, we describe the multi-agent simulation and Netlogo programming environment. In the third section, we discuss and compare the characteristics of simulated solutions for the proposed models and its consequences in the synchronization of the circadian rhythm. In the last section, we present our conclusions and final considerations of this contribution.

\section{Theorectical References}

\subsection{Biological Rhytms}

Biological rhythms are mechanisms capable to determinate behavioral rhythms of the organism. In an imperceptible way they regulate most of the activities of the human being. We will analyze in this work two rhythms: the circadian rhythm and the homeostatic rhythm.

\subsubsection{Circadian Rhythm}

The circadian rhythm regulates important functions of the human body, such as cell renewal and digestion in vigil. It has a 24 -hour periodic cycle regulated by a biological clock.

The clock that monitors the activity cycle of the human body is located, specifically, in the suprachiasmatic nucleus. This nucleus is connected to other parts of the brain and it works as a pacemaker that regulates the rhythm, e.g., (Chiesa, 2005) and references there.

Situations and acts that do not occur daily but that arise over the days effect in circadian synchronization. The factors are diverse, among them unexpected situations as accidents, travels, or even daylight saving time. On of our objective in this study is to share some light in the influence of pain as a source of circadian rhythm desynchronization. Furthermore, an important result of this study to understood in with conditions the circadian rhythm returns into normality conditions after an external influence, i.e., the synchronization.

\subsubsection{Homeostatic Rhythm}

The homeostatic rhythm, defined as sleep pressure, is due to sleep accumulated during the day and decreases during the night.

Excessive hours of sleep causes serious impairments in physical and cognitive performance. However, complex cognitive activities and those with a substantial monitoring component suffer from reduced efficiency of cognitive processing due to sleep deprivation (Ellenbogen, 2005), (Horne et al., 1983). In this way, the absence of sleep affects the efficiency of these tasks.

An important fact about sleep is that we are able to hear the clues of the body and stay awake, even when we are tired, or even get up before having adequate rest. It is important to emphasize that for human survival, being able to adapt to sleep patterns is extremely important, since many abuse the capacity to live and privatize the quality of sleep.

Stefânia da Silveira Glaeser, Angélica Theis dos Santos, Adriano De Cezaro, Catia Maria dos Santos Machado, and Diana Francisca Adamatti

Modeling of Circadian Rhythm under influence of Pain: an approach based on Multi-agent Simulation
ADCAIJ: Advances in Distributed Computing and Artificial Intelligence Journal Regular Issue, Vol. 7 N. 2 (2018), 13 -25 eISSN: 2255-2863 - http://adcaij.usal.es Ediciones Universidad de Salamanca - CC BY NC DC 


\subsubsection{Synchronization}

Each person's biological clock is synchronized according to her/his daytime activities. When synchronized, the internal timetable is correct. However, many factors can perturb the internal regulation, such as during a disruption of our usual schedule given by jet lag, shift schedules given by insomnia our pain have great clinical and economic importance. The question is how our body can get back to his normal activity? For that, an the adjustment mechanisms that allow synchronization are necessary. This synchronization is performed by the adjustment phenomenon, called "entrainment". This external cause that commands the adjustment, is called "zeitgeber". Zeitgebers are the biological clock synchronizes. In this way, the circadian and homeostatic rhythms are synchronized by the zeitgebers, and they are always connected by a type of pacemaker (Borbély et al., 1984), (Borbely and Achermann, 1999).

We can quantify the synchronization effect by think in the sleep-wake and any other pacemaker as coupled oscillators with his one internal frequencies. If the coupled size is larger than the size of the frequencies differences, then we have the synchronization of the circadian rhythm. On the other hand, we might expect that desynchronization occurs.

\subsection{Mathematical Models}

Along the years, a number of mathematical models were proposed to understood the connections between sleepwake cycle and various circadian pacemaker as temperature and neuroendocrine, e.g., (Borbély et al., 1984;

Strogatz, 2013; Chiesa, 2005)and references there.

In (Strogatz, 2013) propose a simple model of the human sleep-wake cycle that might not by to be realistic in detail, but only to capture the key features of the experimental phenomena, mainly the synchronization an desynchronization. The Strogatz simple model assume that the dynamics of the phase model is given by the coupled system of nonlinear ordinary differential equations

$$
\begin{aligned}
& \dot{\theta}_{1}(t)=\omega_{1}-B \cos \left(2 \pi\left(\theta_{2}(t)-\theta_{1}(t)\right)\right) \\
& \dot{\theta}_{2}(t)=\omega_{2}+A \cos \left(2 \pi\left(\theta_{1}(t)-\theta_{2}(t)\right)\right)
\end{aligned}
$$

where the oscillator $\theta_{1}$ represents the body temperature cycle, the oscillator $\theta_{2}$ represents the sleep-wake cycle. The $\cdot$ represent the derivative and $\omega_{1}, \omega_{2}$, are the intrinsic frequencies and the non-negative parameters $A, B$, are the coupling forces responsible to find the influence that one oscillator exert on the others (see the two left oscillators in figure 1 as an interpretation for the model). One of the main advantage of the Strogatz model (1)(2) is that it can be solved analytically e.g., (Glaeser, 2016; Strogatz, 2013).

In this contribution, we propose the introduction of the pain as a new part that acts as an external rhythm in the model governed by the sleep-wake and body temperature cycles (1)-(2), as proposed in Glaeser (Glaeser, 2016). It consist in the introduction of a new nonlinear coupled oscillator $\theta_{3}$, representing the pain, in the model proposed by Stogatz in (Strogatz, 2013), as presented in Figure 1, Moreover, after the ideas presented in Figure 1 , we shall assume that

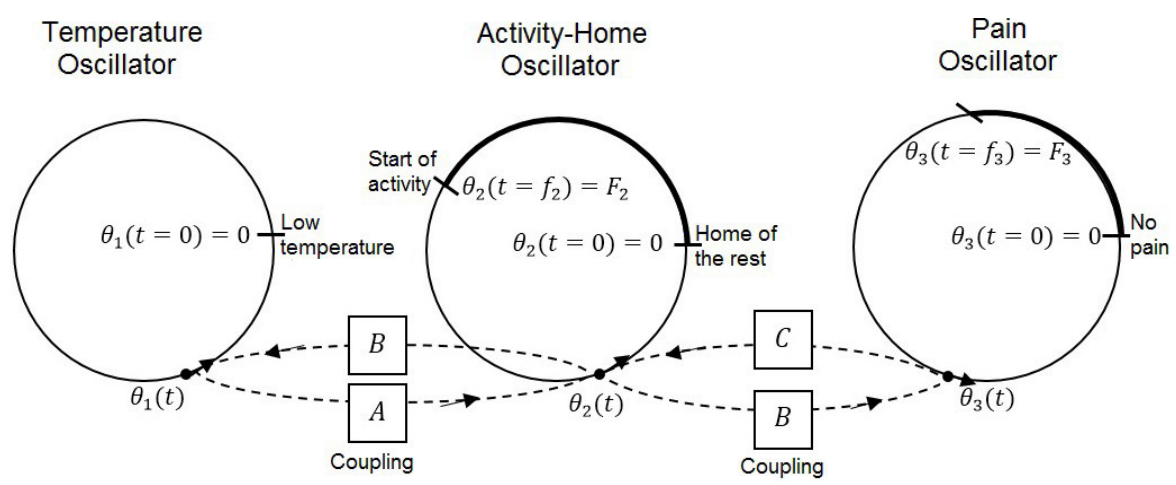

Figure 1: Model Circadian Rhythm

Stefânia da Silveira Glaeser, Angélica Theis dos Santos, Adriano De Cezaro, Catia Maria dos Santos Machado, and Diana Francisca Adamatti

Modeling of Circadian Rhythm under influence of Pain: an approach based on Multi-agent Simulation

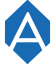

ADCAIJ: Advances in Distributed Computing and Artificial Intelligence Journal Regular Issue, Vol. 7 N. 2 (2018), 13 -25 elSSN: 2255-2863 - http://adcaij.usal.es Ediciones Universidad de Salamanca - CC BY NC DC 
the pain first acts interfering in the sleep-activity rhythm, and after in the body temperature. Others alternatives was presented in (Glaeser, 2016).

Therefore, for this simple model, we assume that the dynamics of the phase model is given by the coupled system of nonlinear ordinary differential equations

$$
\begin{aligned}
& \dot{\Theta}_{12}(t)=\Omega_{1}-C \cos \left(2 \pi\left(\theta_{3}(t)-\Theta_{12}(t)\right)\right) \\
& \dot{\theta}_{3}(t)=\omega_{3}+D \cos \left(2 \pi\left(\Theta_{12}(t)-\theta_{3}(t)\right)\right),
\end{aligned}
$$

where $\omega_{1}, \omega_{2}, \Omega_{1}$ and $\omega_{3}$ are the intrinsic frequencies and the non-negative parameters $C$ and $D$ are the coupling forces responsible to find the influence that one oscillator exert on the others. Note that, from the Figure 1, that we build a new nonlinear coupled oscillator $\Theta_{12}$ that shall be seem as an oscillator formed by $\theta_{1}$ and $\theta_{2}$, respectively. Therefore, we need to interpret (1)-(4) as the influence of the pain oscillator $\theta_{3}$ in the coupled oscillator $\Theta_{12}$ that we consider here as the principal oscillator, for with the influences of pain acts first in the temperature cycle. The quantities $F_{2}$ and $F_{3}$ are the time part of the day where there is no activities and no pain respectively.

With the standard theory of differential equations, we can prove the existence, uniqueness and dependence of the initial conditions and parameters for the systems of equations (1)-(2) and (3) (4). But, in some simple situations, we can derive analytic solutions for the aforementioned equations.

The model (1)-(4) is much more involved that the one proposed by Strogatz, since it has an extra oscillator to be consider. Nevertheless, it can be solved analytically in some situations. In to see that, define

$$
\begin{aligned}
& \psi_{1}(t)=\theta_{1}(t)-\theta_{2}(t) . \\
& \psi_{2}(t)=\Theta_{12}(t)-\theta_{3}(t) .
\end{aligned}
$$

where $\Omega_{1}=\omega_{1}-\omega_{2}, D_{1}=A+B, \Omega_{2}=\Omega_{1}-\omega_{3}=\omega_{1}-\omega_{2}-\omega_{3}$ and $D_{2}=D_{1}+C=A+B+C$, we see that, by taking the derivative with relation to $t$ in (??) and (??) and by substitution of the resulting quantities in (1) and (4), we have

$$
\begin{aligned}
& \dot{\psi}_{1}(t)=\Omega_{1}-D_{1} \cos \left(2 \pi \psi_{1}(t)\right) \\
& \dot{\psi}_{2}(t)=\Omega_{2}-D_{2} \cos \left(2 \pi \psi_{2}(t)\right)
\end{aligned}
$$

Note that, in (7) and (8), $\mathrm{W}_{1}$ is the intrinsic frequencies differences for the principal oscillator, $D_{1}$ is the total coupling forces in this oscillator, $\Omega_{2}$ is the intrinsic frequencies difference between the principal oscillator and $\theta_{3}(t)$ and finely $D_{2}$ is the total coupling forces of the system.

The system may be synchronized or desynchronized. As explained before, synchronization occurs when $D_{1}>\left|\Omega_{1}\right|$ and $D_{2}>\left|\Omega_{2}\right|$. Hence, we define

$$
k_{1}:=\left|\frac{D_{1}}{\Omega_{1}}\right|>1 \quad \text { e } \quad k_{2}:=\left|\frac{D_{2}}{\Omega_{2}}\right|>1 .
$$

as the synchronized stage of the oscillator. The opposite case is the desynchronized stage.

The derivation of synchronized and desynchronized solutions can be seen in Glaeser (Glaeser, 2016).

\subsubsection{Borbély Model}

(Borbély et al., 1984) define that sleep is seen as a process of recovery from fatigue, arising from the vigil in the previous day. Sleep is regulated by two processes, the circadian rhythm and homeostatic rhythm. The wakesleep cycle is defined as the result of the interaction of these two processes.

This model analyzes only a pacemaker, located in the suprachiasmatic nucleus of the hypothalamus, which functions through the mechanisms of zeitgebers. The sleep-wake cycle is drawn by the retinohypothalamic tract, generating numerous physiological circadian oscillations. To explain these oscillations, we have two thresholds (S-Thresholds) H and L, where S (interaction time to generate the circadian rhythm ( and homeostatic rhythm) increases during vigil until reaching $\mathrm{H}$, where is the maximum height for the onset of sleep. In this

Stefânia da Silveira Glaeser, Angélica Theis dos Santos, Adriano De Cezaro, Catia Maria dos Santos Machado, and Diana Francisca Adamatti

Modeling of Circadian Rhythm under influence of Pain: an approach based on Multi-agent Simulation
ADCAIJ: Advances in Distributed Computing and Artificial Intelligence Journal Regular Issue, Vol. 7 N. 2 (2018), 13 -25 eISSN: 2255-2863 - http://adcaij.usal.es Ediciones Universidad de Salamanca - CC BY NC DC 
way, S-Thresholds reduces during sleep until it reaches $\mathrm{L}$ that represents the point of sleep ends. We assume that $\mathrm{S}$-Thresholds decreases during sleep and increases during vigil.

In this way, (Borbély et al., 1984)developed the mathematical model of the two processes, which was improved by (Borbely and Achermann, 1999). After, (Skeldon, 2014) developed it in multi-agent based simulation.

The Homeostatic Rhythm is presented in equation (9).

$$
S_{t}=\left\{\begin{array}{c}
\left.d S_{t-1} ; d=\frac{-\Delta t}{\tau d}(\text { sleep })\right) \\
1-r\left(1-S_{t-1}\right) ; r=\frac{-\Delta t}{\tau d}(\text { wake })
\end{array}\right.
$$

The Circadian Rhythm is presented in equation (10).

$$
C=A\left\{\begin{array}{c}
0.97 \sin \left[\omega\left(t-t_{0}\right)\right]+0.22 \sin \left[2 \omega\left(t-t_{0}\right)\right]+0.07 \sin \left[3 \omega\left(t-t_{0}\right)\right]+ \\
0.03 \sin \left[4 \omega\left(t-t_{0}\right)\right]+0.001 \sin \left[5 \omega\left(t-t_{0}\right)\right] \\
\omega=\frac{2 \pi}{\tau}
\end{array}\right.
$$

All the parameters presented in the equation (9) and (10) were defined by (Borbély et al., 1984), based on data obtained from healthy adults that participated to sleep-wake programs. These people have been exposed to various efforts and reinforcements in their daily environments that help the sleep-wake process to be synchronized.

\subsection{Multiagent Simulation}

Researches developed in Multiagent Systems (MAS) search for models and artifacts that coordinate activities of a group of agents, who must work together. The work in MAS is not only related to the system community or artificial intelligence, it is an interdisciplinary nature, that transits between various areas of knowledge.

Computer simulation is used to study the properties of the mathematical model and have more knowledge in the dynamic process, which in some cases would not be convenient to model with only standard mathematical techniques.

According to Bordini (Bordini et al., 2001)a set of MAS has the main perspective of organizing mechanisms to create computer systems from autonomous software agents that interact side by side with an environment shared by all agents of a society.

The MAS belong to a set of independent agents that organize themselves in the same environment, collaborating each other for a specific purpose (Woolridge, 2001). The agents that form the MAS are computational characters, located in an environment where they are able to perform scripts defined directly or indirectly by the user (Rezende, 2003; Wooldridge and Jennings, 1994).

\subsubsection{Netlogo}

The Netlogo (Wilensky, 1999) is a free software that uses the language $L O G O$ used to develop simulations and derive opinion.

The initial interface of Netlogo is easy to access and manipulate containing information about the model (Info aba) and access to the code (Code aba).

\section{Simulation and Analysis}

We use as basis for computational simulation in the Netlogo the model of Skeldon (Skeldon, 2014.), with the parameters defined by Borbély and presented in Section 2.2.1. For the implementation of novelty in modeling, i.e., for theimplementation of pain as an external effect to circadian and homeostatic rhythms, we use Glaeser's (Glaeser, 2016) studies in the Strogatz model presented in section 2.2.

Stefânia da Silveira Glaeser, Angélica Theis dos Santos, Adriano De Cezaro, Catia Maria dos Santos Machado, and Diana Francisca Adamatti

Modeling of Circadian Rhythm under influence of Pain: an approach based on Multi-agent Simulation
ADCAIJ: Advances in Distributed Computing and Artificial Intelligence Journal Regular Issue, Vol. 7 N. 2 (2018), 13 -25 elSSN: 2255-2863 - http://adcaij.usal.es Ediciones Universidad de Salamanca - CC BY NC DC 


\subsection{Simulation of the Circadian Rhythm Influence of Pain}

In Figure 2, highlighted by the pink rectangle, we can see the inplementation of pain in the Skeldon model. We can also observe the complete interface developed in NetLogo with all variables of the model.

In order to analyze the model, we interviewed a 24-year-old man who presented pain variations during the study period. The young man was questioned for information of 21 days, which will be grouped in three analyzes of 7 days.

Figure 2 describes the first week of observation of this young man. In this period the biological clock was "on", i.e., what defined the characteristic of the circadian rhythm were the "zeitgeber" synchronizers. Therefore, there was not an external or internal factor that intervened in the synchronization of circadian and homeostatic rhythm, not even the pain.

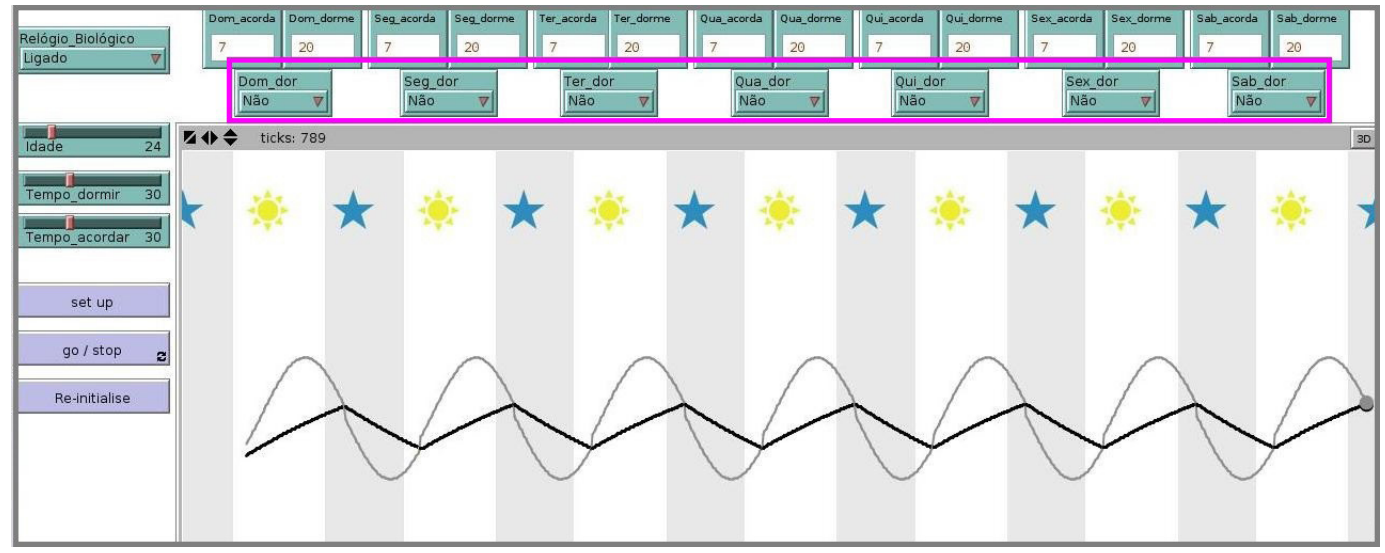

Figure 2: Influence of pain and First Week

From Figure 2, we note that the spaces for the daily wake and sleep times were defined as the times at dawn and dusk, respectively, to better visualize the day and night periods, even if the biological clock is " on " such times do not influence the circadian rhythm. Therefore, the moments in which the respondent fell asleep and woke up are determined by the meeting of the two curves. The time required to sleep and wake up, i.e., a 30-minute sleep fast.

Note also that the gray curve describes the circadian rhythm, it oscillates from top to bottom almost evenly. The black curve describes sleep pressure that increases when the person is awake and decreases when the person is asleep.

Second week of observation the young man did not present evidence of pain, but on two days of the week, Saturday and Sunday, he did not sleep and woke up at the same time of the other days. In addition, the biological clock is "off". Figure 3 describes the embodiment of rhythms.

Stefânia da Silveira Glaeser, Angélica Theis dos Santos, Adriano De Cezaro, Catia Maria dos Santos Machado, and Diana Francisca Adamatti

Modeling of Circadian Rhythm under influence of Pain: an approach based on Multi-agent Simulation
ADCAIJ: Advances in Distributed Computing and Artificial Intelligence Journal Regular Issue, Vol. 7 N. 2 (2018), 13 -25 elSSN: 2255-2863 - http://adcaij.usal.es Ediciones Universidad de Salamanca - CC BY NC DC 


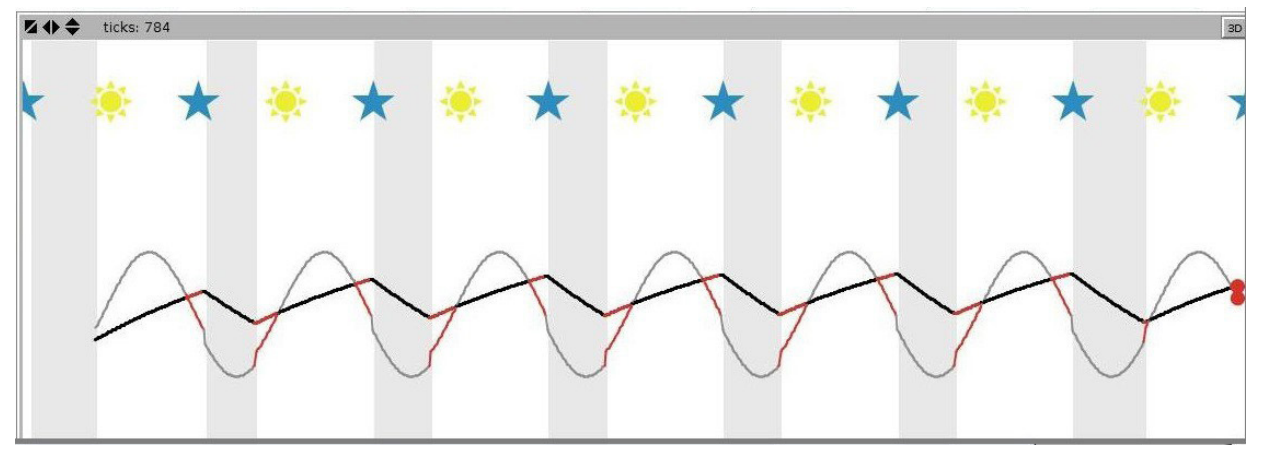

Figure 3: Second Week of Analysis

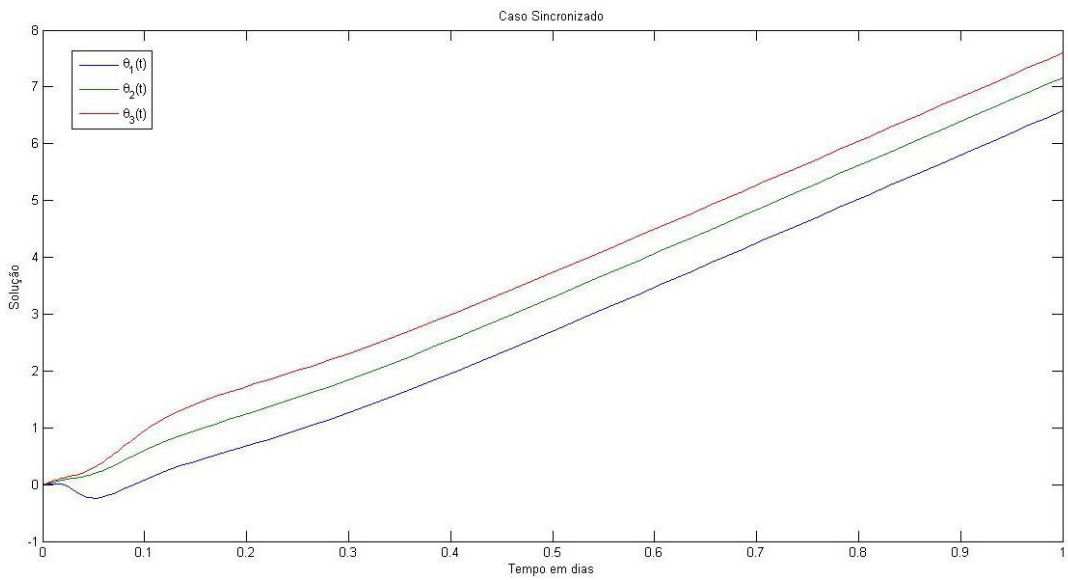

Figure 4: Synchronized Case of Strogatz Model

Let us note from Figure 3 that the moments in which the interviewee falls asleep and wake up are determined by the gray and white parts of the image. Let us also see that in this graph we have red parts along the curves, these correspond to the times in which the biological clock, or the zeitgeber, indicates that you really want to be asleep, but you are awake, so even sleeping 8 hours or more by age, there is a "lack" of sleep. Even so, we were able to see the synchronization of the system.

Looking at Figure 4, we can now see the chart that describes the Strogatz model with the influence of pain. Such curves represent a characteristic of the circadian rhythm. This characteristic defines the oscillation of the rhythms of Figure 3. We can see that from a small time interval the curves present the same pattern, in other words, the circadian rhythm is synchronized.

In the third week of observation, presented by Figure 5, the young man reported evidence of pain on Monday, Tuesday and Friday. Due to the pain, the times of falling asleep and waking were evidently affected, as well as the number of times. In this way, we chose not to change the sleeping and waking hours, in order to better visualize the rhythm of the rhythms as well as because we can not clearly describe the times and the number of times he fell asleep and woke up. Another factor that was altered by the presence of pain was the time needed to sleep and wake up, now being 60 minutes.

Stefânia da Silveira Glaeser, Angélica Theis dos Santos, Adriano De Cezaro, Catia Maria dos Santos Machado, and Diana Francisca Adamatti

Modeling of Circadian Rhythm under influence of Pain: an approach based on Multi-agent Simulation
ADCAIJ: Advances in Distributed Computing and Artificial Intelligence Journal Regular Issue, Vol. 7 N. 2 (2018), 13 -25 elSSN: 2255-2863 - http://adcaij.usal.es Ediciones Universidad de Salamanca - CC BY NC DC 


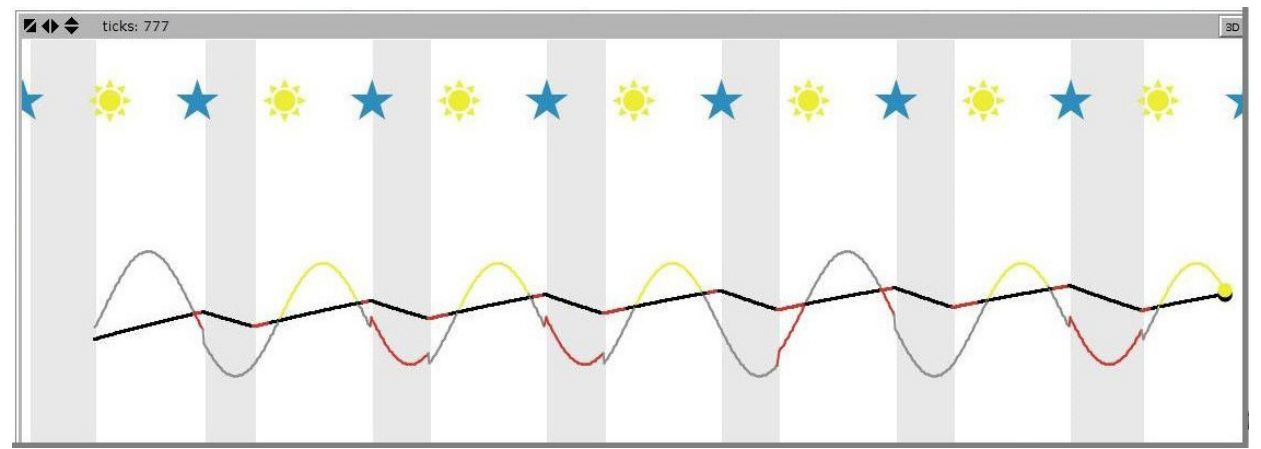

Figure 5: Third Week of Analysis

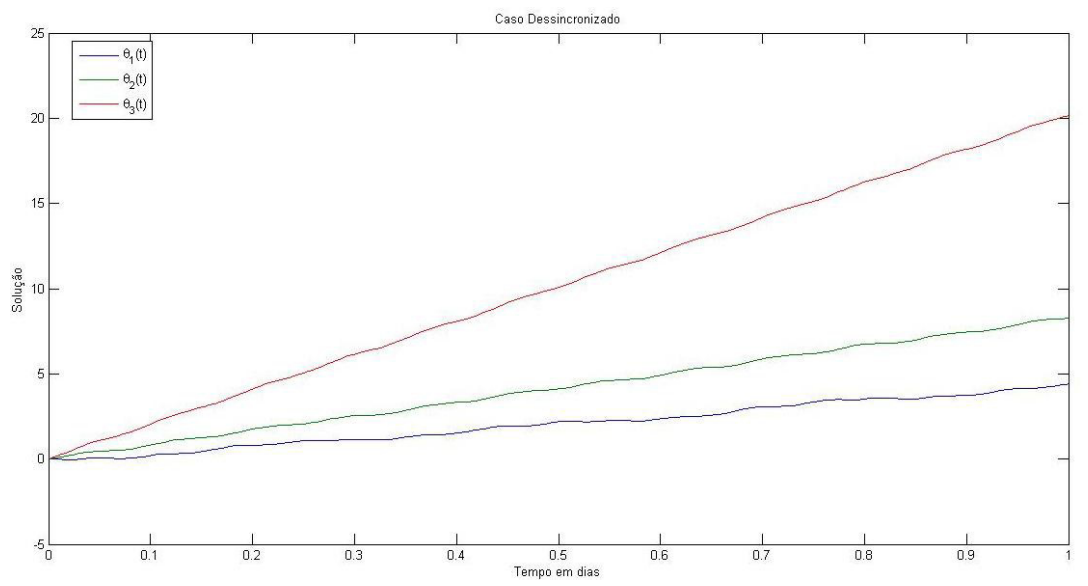

Figure 6: Dessynchronized Case of Strogatz Model

We note in the Figure 5 parts in red on both as curve, the difference here is that these parts are not in period, not well awake, is at the moment, but is awake at the moment, indicate as a moment of rest. In addition, we can see yellow parts in the circadian rhythm curve, these correspond to the times when the body's biological clock is saying that the person must be awake, and is still sitting trying to sleep, note that these parts refer to the moment daytime

And finally, by the Figure 6, we can see the curves describing the behavior of the rest-activity, body temperature and pain cycles of the modified Strogatz model and satisfying the desynchronization definitions for $K 1$ and K 2. These curves define a characteristic of the circadian rhythm. Note that, unlike the previous situation, we can not see a pattern in the behavior of these curves. In other words, the circadian rhythm is desynchronized and such characteristics define the oscillation of the rhythms of Figure 5. Therefore, on days when there is evidence of pain the circadian rhythm does not present synchronization characteristics.

Finally, still analyzing the third week of research, note that on Wednesday there is no evidence of pain, but in the daytime part of the day the person is awake and still try to sleep, this occurs because it takes a period for the system to return to synchronization.

Through these analyzes, we can observe that the modifications of Glaeser in the model of Strogatz complement the simulations of Skeldon in NetoLogo. Note that Skeldon, from Borbély's parameters, uses a multiagent approach that mimics circadian rhythm behavior. However, Glaeser brings a mathematical model that generates characteristics of the synchronization or not of the circadian rhythm under the influence of pain.

In this way, we can observe that on the days when there was no evidence of pain, with the biological clock directly influencing the biological rhythms or not, the system presented synchronization characteristics and on the days that there was evidence of pain the system presented desynchronization characteristics. Also, we can

Stefânia da Silveira Glaeser, Angélica Theis dos Santos, Adriano De Cezaro, Catia Maria dos Santos Machado, and Diana Francisca Adamatti

Modeling of Circadian Rhythm under influence of Pain: an approach based on Multi-agent Simulation
ADCAIJ: Advances in Distributed Computing and Artificial Intelligence Journal Regular Issue, Vol. 7 N. 2 (2018), 13 -25 elSSN: 2255-2863 - http://adcaij.usal.es Ediciones Universidad de Salamanca - CC BY NC DC 
observe that the changes made in the Skeldon code only interfere with the system if there is pain, so we do not affect the veracity of the original program.

\section{Final Considerations}

The biological value of the circadian rhythm is of fundamental importance to any living being. All beings are determined to sleep sometime in the 24 hour period, whether in the morning or evening.

Netlogo, a multiagent simulation environment, is an interdisciplinary tool. The use of it allows an easy learning by visualizing in real time the sleep wake cycle and sleep pressure, which can be influenced by several factors. Pain is one of these factors, as it directly affects the synchronization of rhythms.

Finally, we can see that the model obtained in the Netlogo does not present in a real way the behavior of the circadian rhythm under the influence of pain, but we seek to the maximum with this simple model to imitate the reality.

\section{Acknowledgements}

We thank the Universidade Federal do Rio Grande (FURG), the Programa de Pós Graduação em Modelagem Computacional (PPGMC) and Coordenação de Aperfeiçoamento de Nível Superior (CAPES).

\section{References}

Borbely, A. A. and Achermann, P., 1999. Sleep homeostasis and models of sleep regulation. Journal of biological rhythms, 14(6):559-570.

Borbély, A. A., Daan, S., and Beersma, D., 1984. Timing of human sleep: recovery process gated by a circadian pacemaker. American Journal of Physiology-Regulatory, Integrative and Comparative Physiology, 246(2):R161-R183.

Bordini, R. H., Vieira, R., and Moreira, Á. F., 2001. Fundamentos de sistemas multiagentes. In Anais do XXI Congresso da Sociedade Brasileira de Computação (SBC2001), volume 2, pages 3-41.

Chiesa, J. J., 2005. Patrones Del Ritmo De Actividad Motora Como Expresión De La Plasticidad Del Sistema Circadiano. Ph.D. thesis, Universitat de Barcelona.

Ellenbogen, J. M., 2005. Cognitive benefits of sleep and their loss due to sleep deprivation. Neurology, 64(7):E25-E27.

Glaeser, S. S., 2016. Modelagem de Sistemas Biológicos: Ritmo Circadiano. Master's thesis, Universidade Federal do Rio Grande.

Horne, J., Anderson, N., and Wilkinson, R., 1983. Effects of sleep deprivation on signal detection measures of vigilance: implications for sleep function. Sleep: Journal of Sleep Research \& Sleep Medicine.

Rezende, S. O., 2003. Sistemas Inteligentes: fundamentos e aplicações. Barueri,SP.

Skeldon, A., 2014. Are you listening to your body clock? (http://personal.maths.surrey.ac.uk/st/A.Skeldon/sleep. html).

Surrey Sleep Research Center. University of Surrey.

Strogatz, S. H., 2013. The mathematical structure of the human sleep-wake cycle, volume 69. Springer Science

\& Business Media.

Wilensky, U., 1999. NetLogo.(ccl. northwestern. edu/netlogo). Center for Connected Learning and Computer Based Modeling, Northwestern University.

Wooldridge, M. J. and Jennings, N. R., 1994. Agent theories, architectures, and languages: a survey. In Intelligent agents, pages $1-39$. Springer.

Woolridge, M. J., 2001. Introduction to Multiagent Systems. John Wiley \& Sons, Inc., New York, NY, USA. ISBN $047149691 \mathrm{X}$.

Stefânia da Silveira Glaeser, Angélica Theis dos Santos, Adriano De Cezaro, Catia Maria dos Santos Machado, and Diana Francisca Adamatti

Modeling of Circadian Rhythm under influence of Pain:

an approach based on Multi-agent Simulation
ADCAIJ: Advances in Distributed Computing and Artificial Intelligence Journal Regular Issue, Vol. 7 N. 2 (2018), 13 -25 elSSN: 2255-2863 - http://adcaij.usal.es Ediciones Universidad de Salamanca - CC BY NC DC 
